\title{
Using the local positioning system based on Pulse Width Modulation for Robot Positioning
}

\author{
*11FATEMEH MASOUDIFAR; ${ }^{2}$ NEKOUI, M A \\ * Islamic Azad University, Science and Research Branch, Tehran, Iran fatememasoudi@gmail.com \\ ${ }^{2}$ Electrical Engineering Dept., K.N.T.University of Technology, Tehran, Iran
}

Keywords: LPS, PWM, IFRD

\begin{abstract}
There are many ways to estimate the location of a moving object. One way is to determine its location using a GPS which calculates longitude and latitude of the object by the data received by the satellites rotating around the earth. However, there are no devices to recover calculated inherent errors and have an appropriate estimated signal. From robots' place, we should correct the errors made by collected data from sensors. A problem happening alternatively in the landmark identifying a method is the similar sharing data. The data obtained should be related to correcting land mark. Even though, some landmarks are likely to be similar. In addition, it is impossible to guarantee sightseeing line among landmarks in some arrangements. The other methods are to utilize radio frequency stations, which are placed around the robot by which we can find the real place of source of measurement error. In non-isolated environments, due to noises in signals using such a system should be investigated. In this PAPER, LPS (local positioning system) and the way it influences interference signals on the simulated system has been studied. A method has been tried to present in order to decrease destructive effects of noise using GA (genetic algorithm). In this way, the LPS modulation method and factors which cause interference signal and noises in the system have been stated. The obtained results have been illustrated by different simulations followed by discussions. @JASEM
\end{abstract}

In the past, it was impossible for people, to be aware of their positions in a wide geographical area. As solution, stars and other natural symbols had been used, to some extent. Nowadays, this problem has been answered by geographical positioning systems. Within robots, their ability of positioning has a great effect on the methods through which they are at work. In many applications in which using robots seems appropriate, capability of positioning is a prerequisite.

There are a couple of methods to estimate a robot's position, one way of precise positioning is the use of the global positioning system (GPS) in which a group of satellites circulating around the earth, calculates the geographical location of the object. The drawback of this method is the necessity of existence of a direct sight to the satellites, which is often impossible for the missions considered to be done within departments or underground by robots where they are not able to receive submitted data from satellites. Even in the outside, barriers caused by departments or trees deteriorate since the communication channel between robot and satellites. Moreover, received data from noisy environments is not reliable. Therefore, for specific applications where the exact position of the matters is of high importance, LPS would be used.

The general operation principles of LPS are similar to GPS except that for GPS, there are at least three satellites as the fixed points of emitting and receiving electromagnetic waves, whereas in LPS, receivers are usually installed on the mobile sets, and receivers are fixed on the ground.
In 2000, Sypniewski (2), succeeded to position objects within departments or other blind-points using radio waves that GPS did not. In multi-path environments, in which we have radio wave reflectors, a weight $s(r)$ will be dedicated to each vector (r) of the workspace. Then, position ( $\mathrm{Rx})$ can be estimated using several methods in which the simplest one is to choose the largest $s(r)$ while being able to position the robot even with existing wave's reflectors. Four accessing antenna, at least is needed. The more antenna, used, the more precise positioning.

In 2002, LPS was used to position a flying robot by M. Parntchkun (3). In the robot, ultrasonic waves had been used instead of electromagnetic waves (which are used in GPS). Based on the difference between the times of receiving ultrasonic waves in each receiver located on the ground. The robot would be positioned.

In this positioning system, four receivers had been used to receive the transmitted waves from flying robot. The distance from each receiver was determined based on the time of the received data from receivers. Knowing the distances obtained and ignoring the noise effects, robot can be positioned exactly. Using the mentioned approach, robot was positioned with an acceptable accuracy.

In 2003, a combination of radio waves and infrared waves was used for positioning by T. Pfeifer (4), which had a high accuracy. Based on magnetic fields of very low frequency, Ene A. Prigge designed a set for positioning of the objects. There is no need to have any direct sight between the object and antenna, 
Using the local positioning system.....

in this method, whose accuracy is of a few centimetres. Magnetic waves are able to penetrate the materials easily. Even in cases where the matter is located in wood or cement, it can be easily positioned by the positioning system.

In 2004, an analysis on the robot positioning system was performed by D. Kurth (7). The method has a high accuracy (the average of $15 \mathrm{~cm}$ over the transverse route) but the high complexity off-line processing is necessary. Fourth, then, considered positioning using a particle filter called Monte Carlo, where. A probability distribution is made based on a series of particles that supposed to be the typical positions of the robot. In case of the primary distribution given as a default, it can be seen that the particle filter does not need any estimation for the primary position.

Finally, positioning problem was considered in such a way that robot absolute measurement is obtained using ground indicators whose positions are not clearly known. The case is called SLAM where a well-known solution in the form of a Kalman Filter is sought. The results are comparable with the Kalman Filter based positioning. For description of noise in measurement of seed, whenever the robot passes a measurement interval, the corresponding error will be considered.

\section{Modelling of the Positioning System}

Short wavelength radio waves having high permittivity let them pass through materials easily. Therefore, they can be used in positioning where there is not a direct line between materials and antenna. In this regard, they are preferable so that ultrasound waves are increasingly used. Using this method has made the related receivers (like the RFID reader) easily accessible and cost-effective. Radio waves produced in the form of a consecutive pulse will be transmitted by robot and received by the ground receiver. Since, robot is not in synchronization with the positioning system located on the ground, travelling time needed for the waves to reach the antennas cannot be calculated and only the differences between these times will be obtained. In order to correspond to these differences to their related pulse signals, they should be coded and modulated. Thus the pulse width modulation has been used.

(Scheme of modulated signal used in the local positioning system)

This signal has two types of data: up-going edge for measuring the difference between the reception time of waves in different antennas and its width, which determines the pulse number to distinguish signals from one another in the antennas. A proposed distribution of the antennas and the receiver is shown in figure 1 .
The frequency of up-going edge $\left(v_{2}=1 / T_{2}\right)$ determines the frequency of positioning, which should be selected based on the accuracy needed in the measurement system. T1 shows the repetition period of pulse width. In a given time interval, this period should be less than as much as twice of the maximum time difference between the signals received in different antennas in order that the corresponding signals in individual antennas will be chosen correctly.

To start, the first period $T_{2}$ should be determined based on the desirable measurement frequency which in our case is $\mathrm{f}=10 \mathrm{MHz}$, with regard to $T_{2}=0.1 \mathrm{~ms}$. Then, maximum distance of a working point of robot from two different antennas should be calculated based on the working geometry of robot and antennas, which will be presented in the following section which is $260 \mathrm{~km}$ in distance.

Knowing that the wave emitting speed is $300 \mathrm{Mm} / \mathrm{s}$, shortest pulse width period is $T_{2}=0.87 \mathrm{Ms}$, which along with the reliability coefficient, it includes 10 pulses.

As it will come in the following, measuring the time difference between reception times of individual pulses in different antennas, robot can be positioned.

Geometry of the robot environment: In this system, radio transmitter and receivers (antennas) are located on robot and ground, respectively. Number of antennas which are non- homogeneously spread over the working area, is 8. Positioning form of the antennas has been shown in figure1.

Phone-waves which are an important factor in system noise have been considered in simulation with noise, as shown in the following figure.

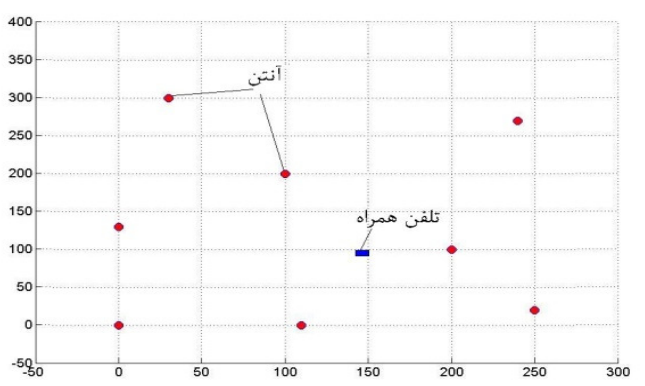

Figure1: a proposed pattern for antenna locations

Robot dynamical simulation: For dynamic modelling, resulted from the variety of robots, the main indices of models including dimension details, inertia, friction, and other dynamic parameter that would be defined for robots, specifically, are not considered here. The robot modelled here, has the 
Using the local positioning system..... capability of moving in two directions. The maximum speed accessible, in this case is $10 \mathrm{~m} / \mathrm{s}$ (V $\max =10 \mathrm{~m} / \mathrm{s}$ ) which is determined by such parameters as maximum operator's power, aerodynamic form of robots, interval doses of frictions. Considering the types of operators, weight and dimension at robots, maximum acceleration of $2 \mathrm{~m} / \mathrm{s}^{2}$ is possible for it. Since robots, nowadays, have the high capability of manoeuvre. In all-directional movement, no limitation has been put on the movement's direction and the corresponding acceleration. Considering the system's inertia and friction, dynamic relation will be as follows:

$$
\begin{aligned}
& \Delta v=a t=\frac{F}{M} t=\frac{u \circ-C \circ V}{M} t=u-C V \\
& V_{2}=(1-C) V_{1}+u \\
& X_{2}=X_{1}+V_{2} T
\end{aligned}
$$

Where $u_{0}$ is the imposed force by operators, $C_{0}$ is friction coefficient $V_{2}$, are robot speeds in the present and previous time steps respectively, and $\mathrm{T}$ is time step.

Considering the measurement period of system with all other parameters which are given approximately, the dynamic relation of the system will be:

$$
\begin{aligned}
& V_{2}=0.95 V_{1}+u \\
& X_{2}=X_{1}+0.1 V_{2}
\end{aligned}
$$

Where, maximum values of $\mathrm{u}$ and $\mathrm{v}$ (robot speed) are $\mathrm{U} \max =0.2$ and $\mathrm{V} \max =10$, respectfully. The control system has been designed for two different cases. In first case robot is to be Positioned in certain location while in the second, it will follow a predetermined route.

Case 1: Positioned, so that the position would be certain by transition vector, here based on the error in location positioning the divined speed in the destination's direction will be obtained and using the robot speed of previous moment, the present speed will be extracted.

Case 2: Moving on a predetermined route: as in case location and speed of robot. Now based on the dynamics of robot, the necessary force for reaching the desired route will be calculated. Here again the error of robot position affects speed, in other words when there are much disturbance in the environment which in turn leads to more error in robot positioning, low speed will be considered for it (by control) so that deviation from the main route will be kept low.

Simulation of waves in transmitter and receiver: In this section, based on the position of robot against antennas signal's reception time in each antenna is calculated. To do so, considering the position first of the robot, its distance from the antenna will be obtained. Then, with regard to the speed of wave distribution, the reception time of wave to each antenna is calculated.
The difference between the obtained times needed for wave to reach the antenna (antenna No 1.) will be chosen as the out put. Until now, only the reception times of signal for antennas in isolating the environment have been derived. To include the interference factors in the measurement system according to paper by Derek kurth (7) noise is simulated as Gaussian Function. These noises can be classified into two groups: first, those ones with similar effects on the received signal as the transmitted signal produced by the electromagnet, i.e. Components of robot and effect of humidity on wave emission speed, are considered as.

Gaussian the second kind of noise are those stemmed from external factors, the main source of this noise is mobile phone .Since electromagnetic waves power is inversely to distance from the source, the closer the antenna to the mobile phones the more noise. Thus it is modelled as Gaussian function proportional to distance from mobile phone.

Positioned modelling using Genetic algorithm: As mentioned above using three antennas and in the absence of noise effect, robot can be positioned exactly at the point where the circle with radius of distance between antenna and robot and it's location at the antenna placement each other. In the presence of noise with some antennas, however, there is no certain answer, in other words circles don't meet each other at the common point. In this system, reception time of waves to the antenna is measured in relation to the given reference antenna (No.1). Using the obtained data from the present and past robot will be positioned through genetic algorithm. With the execution of the program, the effect of various external factors on the mentioned system would be analyzed and parameters of the Genetic Algorithm will be optimized. In the following, this algorithm will be reviewed in detail. The total number of chromosomes is 10. Each chromosome is defined as $(x, y)$, where $x, y$ indicate the estimate location of the robot this number should be located at the working place of the robot. That is, through all steps, location of coma some will be checked and in thus the case it was not in the desired area. It Primary chromosome is produced randomly around the previous location at robot. In this way convergence rate of the algorithm will be risen, nevertheless, they may fall into the local minims that mean this system is not able to position the robot. In order to solve the problem the radius over which the primary chromosomes were located around the previous location of the robot should be chosen so that the convergence high enough in the local minimums will be avoided.

As mentioned above, maximum speed of robot is 10 $\mathrm{m} / \mathrm{s}$ up to 10 meters it can be far from its earlier location, since there are some errors in robot positioning, in order for the program to be convergent 
Using the local positioning system.....

of 30meters radius with its centre at the ear lire location of robot will be selected as the area of primary chromosome production. Cost function is combining of parts: one for the data extracted of the present time the other for the previous data and the control's effort. Thanks to the use of the prior information the latter improves the accuracy of positioning and reduces the effect of stochastic noise on system .However this factor should be passed sophisticatedly and relative to the present measurement error .So that to avoid the accumulation of permanent error in robot positioning, these factor will be analyzed in the upcoming section. As mentioned before robot can be positioned using the difference between the reception times of wave in reaching antennas. To do so, we have:

$$
\begin{gathered}
x^{2}+y^{2}=y_{1}{ }^{2}=\left(t_{1 . s}\right)^{2} \\
(x-x i)^{2}+\left(y-y_{i}\right)^{2}=r^{2}=r i^{2}=\left(\left(t_{1}+\Delta t_{1)} \cdot s\right)^{2}\right.
\end{gathered}
$$

Where $(\mathrm{x}, \mathrm{y})$ is the location of robot $(\mathrm{Xi}, \mathrm{Yi})$ location of the $\mathrm{i}$ time for robot, wave to reach the antenna 1 (reference antenna) and $\Delta t_{i}$ time is the travelling time of wave between this antenna with respect to the reference antenna, siantenna, s operation is not synchronized with ground positioning system $t_{1}$, is eliminating the $T_{1}$ from the governing equation on system usingthe system leads to:

$$
(x-x i)^{2}+\left(y-y_{i}\right)^{2}-\left(\sqrt{\left.\left.x^{2}+y^{2}+\Delta t_{1}\right) s\right)=0}\right.
$$

This equation holds for isolate environment that is in case of noise it would not be held and we have:

$$
(x-x i)^{2}+\left(y-y_{i}\right)^{2}-\left(\sqrt{\left.\left.x^{2}+y^{2}+\Delta t_{1}\right) s\right)=\Delta e_{i}}\right.
$$

Error of these equations will be used for the calculation of the first part of cost function of the Genetic Algorithm program i.e. the first factor on cost function would be the sum of difference in (5)

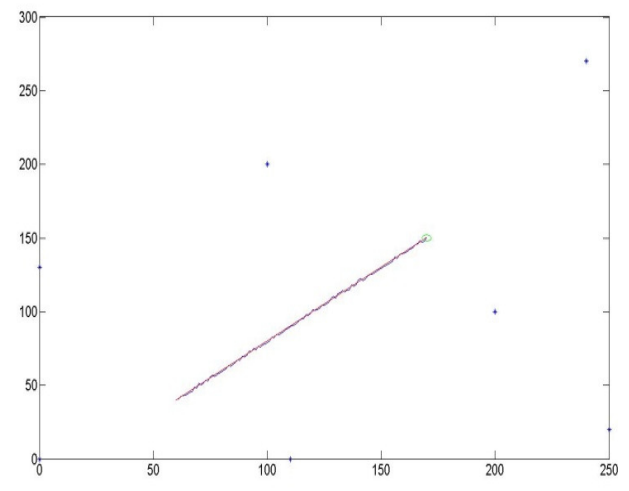

which is as follows:

Figure1: the robot trajectory in the isolated simulation

$$
e_{1}=\sum_{r_{1}}^{n} \frac{\Delta_{1}}{r_{1}}=\sum_{i=1}^{n} \frac{\left(x-x_{i}\right)^{2}+\left(y-y_{i}\right)^{2}-\left(\sqrt{\left.x^{2}+y^{2}+\Delta_{1} s\right)}\right)^{2}}{\left(x-x_{i}\right)^{2}+\left(y-y_{i}\right)^{2}}
$$

The second factor of cost function is the location positioned by robot, which can be obtained using the previous location in which error can be calculated Xs (estimate location by robot) is calculated based on the location of robot and control effect in the previous step time. To do so, the following dynamical equation of the robot will be used:

$$
\begin{aligned}
& V_{2}=0.95 V_{1}+u \\
& X_{2}=X_{1}+0.1 V_{2}
\end{aligned}
$$

Using the previous location and control effect done Xs will be obtained as follows:

$$
X_{s}=X_{1}+0.1 V_{2}
$$

Then the difference between the new estimate and measured location will build the second factor of cost function as mentioned before, this factor increases the measurement accuracy and decreases the effects of stochastic noises on the system. It must be imposed in a way so that permanent the accumulation of error and in turn, permanent error in measuring the robot location. Bearing this in mind, it must be relative to the pervious measurement error so that the more the present time error (ei) than the previous time and the more effects of stochastic noise decreases. On the other hand, the smaller the present time error than the previous time the less effect of a factor and precious from the error to be accumulated therefore final cost function will be defined as follows:

$$
E=e_{1}+C \frac{e_{1}}{E_{0}}\left|X-X_{s}\right|
$$

Where $\mathrm{Xs}$ is the estimate location by $\operatorname{robot} \boldsymbol{E}^{2}$. Positioningprevious step and $\mathrm{C}$ is a constant, coefficient that takes the effect of data from previous step into account: that is when it increases, this effect increases vice versa to have the optimal coefficient $\mathrm{C}$ error of positioning relative to its given location will be evaluated for various $\mathrm{c}$. As it is mentioned in the following chapter the optimal $\mathrm{C}=$ 0.4. After arranging the chromosomes and selecting the better ones, the best chromo some as elite willthe elite ted by other ones to reproduce the new generation, to this end the following relation is used:

$$
x_{t}^{\text {child }}=\rho X_{e l}+(1-\rho) X_{1}
$$

Where $\mathrm{Xi}$ child is the child chromosome, $\mathrm{X}$ el is the elite and $\mathrm{P}$ is a random figure. Finally, this child will be compared with other chromosomes, if it is different from the others it will be selected as new chromosome to avoid. Falling into the local minims a chromosome other than the elite will be selected and will be selected randomly in the working environment of the robot. From now on, these steps will be repeated until the convergence condition to be satisfied. This condition, is considered to be the simultaneity convergence of the elite and chromosome variance in order to verify the modelled 
Using the local positioning system..... positioning system first, it should be simulated in an isolated environment.

Robot trajectory in the isolated simulation is shown in this figure. The trajectory is a straight line pointed toward the target in fig 2. Error of positioning relative to the given location is drawn versus the time stop of the robot move. It can be seen that primarily estimated locations are accurate and of high convergence since the certainty of the point's coordination. However, as time passes by, the estimate location would rate differently from the actual one partially; it stems from the large convergence interval: as this interval decreases, the accuracy in the system the increases but the number of step convergence increases, too.

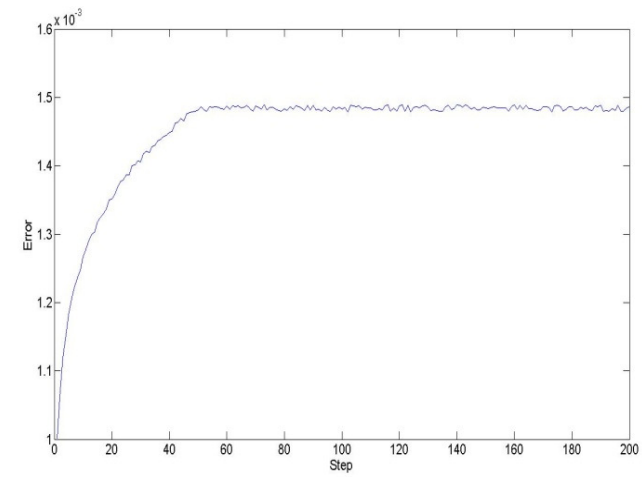

Figure2: error of positioning in the isolated simulation)

Error of positioning in the isolated simulation now after the modelling verification effect of noise on the positioning system will be analyzed .To this goal, first the system parameters will be optimized and then having a convergent performance, effects of different noise will be investigated. For the purpose of investigation of the cost function of the genetic algorithm, robot moving toward a given destination would be simulated two times with (effects of the previous data) and without the send part of cost function included .The obtained results are depicted in figure 3 and figure 4 :.

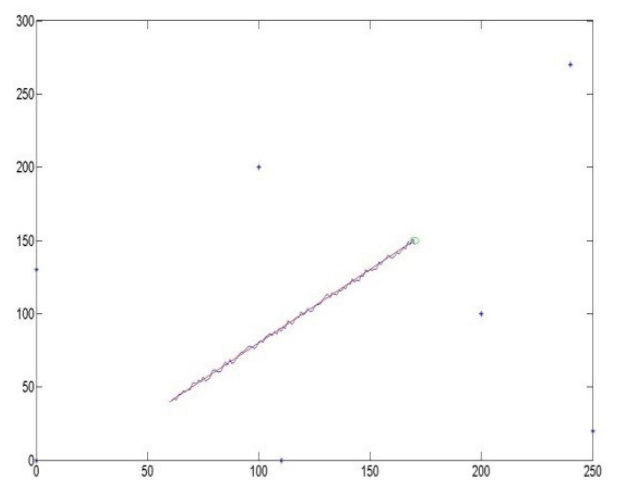

FATEMEH MASOUDIFAR; ${ }^{2}$ NEKOUI, $M$ A
Figure3-2: the robot trajectory with considering the effects of the previous data)

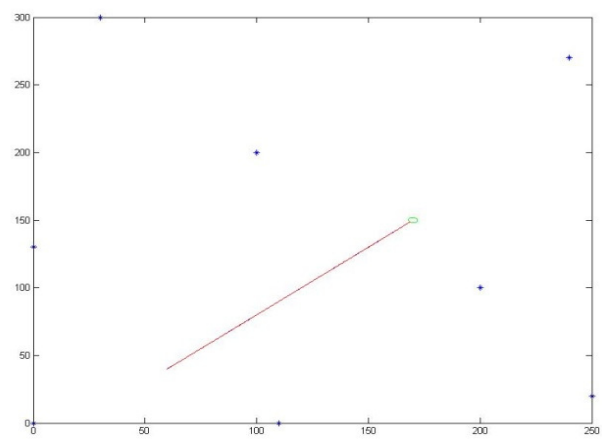

Figure 4: the robot trajectory with considering the effects of the previous data)

It can be seen that because of random behaviour of noise, there are many fluctuations in the positioning in the first simulation (ignoring the second past of cost function).

Considering the second part and presenting the previous data into the positioning. These fluctuations will decrease. In other words, this factor imposes integration on the obtained data which along with the random behaviour of noise in decreases the inverse effect of noise and the fluctuations of the system. As the mentioned before, effect of each part of cost function should be determined in a way to decrease the oscillatory behaviour of the system in one hand and prevents the accumulation of error, on the other hand, to do so this error from the previous stages will be used as a parameter to determine the correctness of the estimated location through dividing this error to the first part of cost function. There would be a demotion fewer parameters that determine the amount the second factor effect to make the performance of the system better.

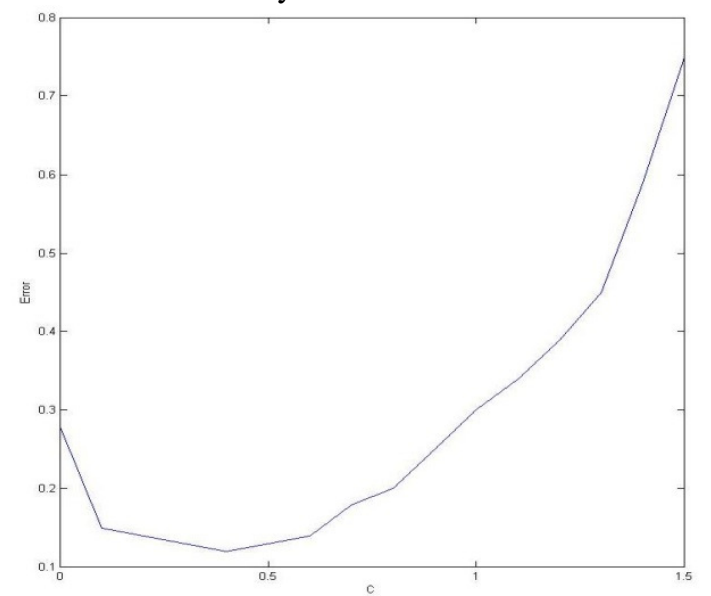

Figure 5: the error of measurement system by constant coefficient C)

Error of robot positioning (difference between the measured location and the actual one) is drawn versus $\mathrm{C}$.It is clear from the figure that for, because of elimination of the second factor so high and decreases to its optimal value at increases the error 
Using the local positioning system.....

and from $\mathrm{c}=1.5$ estimated location of the robot will be separated from the actual one with a deviation input of destination.

When the performance of positioning system is optimized, effect of noise will be analyzed. This will be done once with a cell phone and the other without it. Results one shown infig. 6

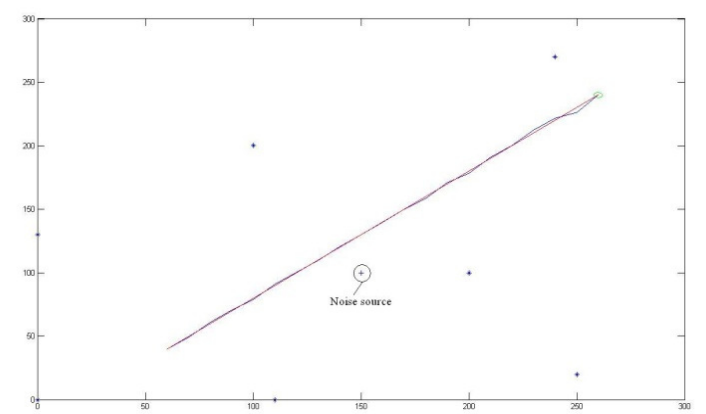

Figur6: the robot trajectory with the existence of external noise source)

Figures 7 and 8 . It can be seen that without cell phone included in the simulation (fig 7).

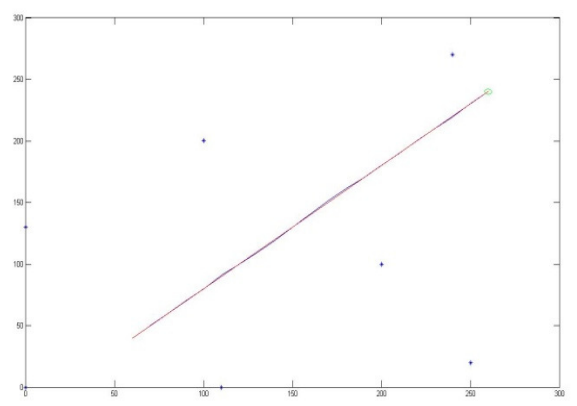

Figure7: the robot trajectory without considering effect of external noise source)

There are some fluctuations over the whole path while with cell phone included when the transmitter (robot) is far from the source of external noise (cell phone) there is more error. This result can be explained so when the cell phone is close to the robot, it presents a random noise that is similar to the main signal which will be received by all antennas were as in the case it is far from the robot in the corresponding noise will be stronger in the closer antennas and the effect of noise will be constantly more some antennas which in turn it causes the accumulation of noises and increases the error which increases the distance between robot and noise source. Now robot performance will be examined in a circular path simulation. The result is shown in (fig 8 ). It is seen that the robot is able to find the path and move along easily. The error of measured location where the robot is far from the source of the external noise (cell phone) is more. In this case error of estimated location of robot is shown in fig 9.

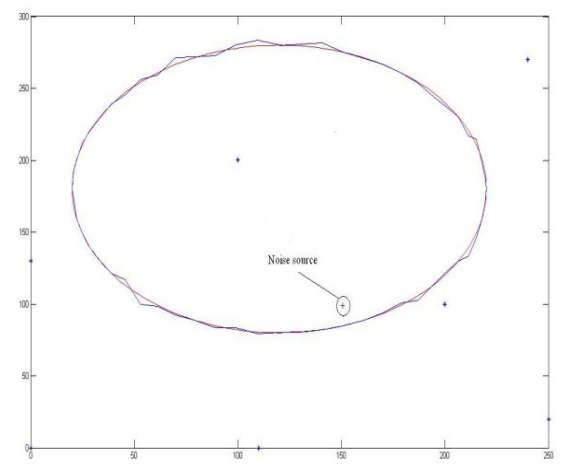

Figure8: the robot trajectory in a circular path simulation)

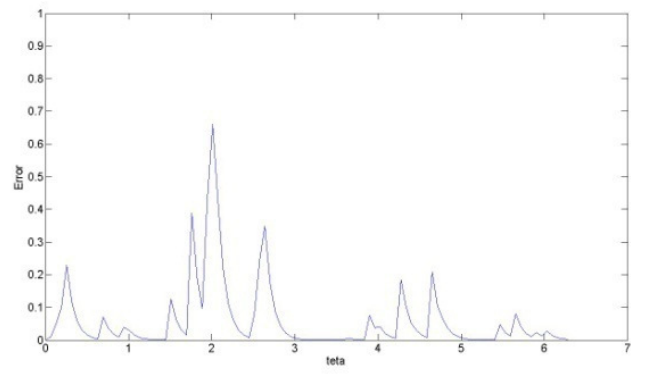

Figure9: the error of measurement system in a circular path simulation. It can be seen that this system can position robot with an error less than6.

Conclusion: Using the radio waves, a local positioning system was modelled and simulated in this paper. through optimizing this system, .It was seen that using the data obtained from the previous time step, system's System's can decreased decrease fluctuations and size of error as well.

Nevertheless, when their effects are too much at work it causes the accumulation of error, permanent error and finally divergence of the system. As a solution this problem effect of previous data was considered relative to the error in the previous step with comparing the simulation results in the proportion of these data in the cost function was considered as $40 \%$ of data obtained up to presented time. Verifying the model simulated and optimizing the corresponding parameter's effect of two types of noise were analyzed. First type of noise was caused by robot and charge imposed on signal speed because of the humidity. It was seen that this noise makes an even fluctuation on the robot's path which does not deviate from the robot from its path and positioning system is still able to position the robot. The other type of noise is external, which is produce by an external source like a cell phone. Simulation results showed that the effects of these external signals when the robot is far from source of noise is more. Finally circular movement of the robot was examined and in This case, system could position the robot with an error less than $0.6 \%$

\section{REFERENCES}


Using the local positioning system.....

1-"Statistical Electronics" Noise Processes in RF Integrated Circuits (Oscillators and Mixers), Donhee Ham, Harvard RF and High-Speed IC \& Quantum Circuits Laboratory

2- "The DSP Algorithms for Locally Deployable RF Tracking System", Josef Sypniewski, SypTech, Co., San Antonio, TX.

3-"Local Positioning System (LPS) for a radio wave-controlled flying robot", Manukid Parntchkun and Soracha kirithaveep , School of Advanced Technologies, Asian Institute of Technology.

4- "Commercial Hybrid IR/RF Local Positioning System”, Tom Pfeifer, Dirk Elias, KiVS 2003, Kommunikation in Verteilten Systemen, Feb 26-28, University of Leipzig, Germany.

5- "A positioning system with no line_of_sight restrictions for cluttered environment," Eric A. Prigge
, submitted to the department of aeronautics of Stanford university in partial fulfilment of the requirements for the degree of doctor of philosophy, August, 2004

6-" Robotics-based location sensing for wireless Ethernet" A. M. Ladd, K. E. Bekris, G. Marceau, A. Rudys, D. S. Wallach, and L. E. Kavraki, In $A C M$ MobiCom, 2002.

7-"Range-Only Robot Localization and SLAM with Radio”, Derek Kurth, CMU-RI-TR-04-29, May 2004.

8- "Simultaneous Calibration of Action and Sensor Models on a Mobile Robot", Daniel Stronger and Peter Stone, Department of Computer Sciences, The University of Texas at Austin, 1 University Station C0500, Austin, Texas 78712-1188. 